\title{
Electro-optic lightning detector
}

\author{
William J. Koshak \\ Global Hydrology \& Climate Center \\ NASA Marshall Space Flight Center \\ Huntsville, Alabama
}

\author{
Richard J. Solakiewicz \\ Department of Mathematics \& Computer Science \\ Chicago State University \\ Chicago, Illinois
}




\begin{abstract}
The design, alignment, calibration, and field deployment of a solid-state lightning detector is described. The primary sensing component of the detector is a potassium dihydrogen phosphate (KDP) electro-optic crystal that is attached in series to a flat plate aluminum antenna; the antenna is exposed to the ambient thundercloud electric field. A semiconductor laser diode $(\lambda=$ $685 \mathrm{~nm}$ ), polarizing optics, and the crystal are arranged in a Pockels cell configuration. Lightning-caused electric field changes are related to small changes in the transmission of laser light through the optical cell. Several hundred lightning electric field change excursions were recorded during five thunderstorms that occurred in the summer of 1998 at the NASA Marshall Space Flight Center (MSFC) in northern Alabama.
\end{abstract}




\section{Introduction}

The most common means for measuring lightning-caused changes in thunderstorm electric fields involves the application of a flat metal plate antenna and a charge integrating apparatus. Because the normal field $E_{n}$ at the surface of an exposed antenna plate satisfies the normal component boundary condition $E_{n}=Q /\left(A \varepsilon_{o}\right)$, where $Q$ is the induced charge, $A$ is the plate area, and $\varepsilon_{0}$ is the permittivity of free space, a change in the thunderstorm field must be accompanied by a change in induced charge. The integrating apparatus used to measure the changes in induced charge normally consists of an electronic integrating circuit composed of an electrometer and having in the feedback loop a resistor $R$ and capacitor $C$ in parallel. The inverting input of the electrometer is attached to the plate and the noninverting input is grounded. Typical $R C$ decay times of the electrometer output are measured in seconds (slow antennas) and from milliseconds to microseconds (fast antennas).

From the point of view of the experimenter, the $R C$ decay time should be made larger than any time variation of interest [1]. For instance, if one is interested in lightning return stroke fields, the $R C$ should be in milliseconds and if interested in total lightning field changes, the $R C$ should be in seconds. The decay assures that the sensor output is nullified before another event occurs; triggering from the "zero line" is an advantage because one can devote the full vertical amplitude resolution and dynamic range of the sensor to the event. The systems described by [2] employed $R C$ values of $4 \mathrm{~s}, 0.3 \mathrm{~ms}$, and $70 \mu \mathrm{s}$ to look at various temporal regimes in the lightning signature.

In the past 20 years, several new scientific and commercial applications of electro-optic technologies have arisen for measuring electric voltages and fields ([3] - [10]), and some of these investigations were specifically directed toward the study of thunderstorm electric fields. 
Electro-optic crystals have offered a convenient and affordable means of controlling the intensity or phase of optical radiation in an ever-expanding number of applications that include high-speed digital communication, optical beam deflection, and spectral tunable filters [11]. Because the crystals respond quickly to applied voltages and no moving parts are required, modulation frequencies of several gigahertz are possible. Liquid crystals have also been used in high speed optical shutter applications, and [12] has used liquid crystals to measure lightning electric field changes.

In the present study, we describe an electro-optic lightning field change detector that was recently developed and deployed in the summer of 1998 at the NASA Marshall Space Flight Center (MSFC) in Huntsville, Alabama. The sensor is an engineering model composed of both fabricated and vendor-supplied components, and the optics were mounted on a small laboratory optical breadboard. The $R C$ decay was adjustable from $1 \mathrm{~s}$ to $1 \mu \mathrm{s}$ and was typically set to a few tens of milliseconds during deployment in order to resolve individual strokes in a lightning flash. The main sensing components are a flat aluminum plate and a long cylindrical, high impedance electro-optic crystal of potassium dihydrogen phosphate (chemically written as $\mathrm{KH}_{2} \mathrm{PO}_{4}$, or "KDP" for brevity); some dielectric and optical properties of KDP are provided in [13]. The details of the design, alignment, calibration, deployment, and data results are discussed below. We begin our discussion with a review of the linear electro-optic effect since it is fundamental to the design characteristics of the lightning detector.

\section{The Linear Electro-Optic Effect}

Friedrich Pockels (1865-1913) was the first to describe the linear electro-optic effect in two early publications ([14], [15]). Figure 1 shows a typical Pockels cell arrangement for an electrooptic modulator. The central component is an electro-optic crystal, KDP. Light from a diode 
laser (input beam) passes through two polarizers, a quarter wave retarder, and the crystal before exiting the optical system. The output beam would typically be measured with a photodetector and transimpedance current amplifier system.

Equations for the transmission of light through an anisotropic medium such as the KDP crystal may be derived from Maxwell's equations and energy conservation. Conductivity $\sigma$ is negligible for the materials we are using at optic frequencies. The electric displacement $\mathbf{D}$ and magnetic induction $\mathbf{B}$ are related through [16]

$$
\nabla \times \mathbf{F}=-\partial \mathbf{B} / \partial t, \nabla \times \mathbf{H}=\partial \mathbf{D} / \partial t+\mathbf{J}, \nabla \cdot \mathbf{D}=\rho, \nabla \cdot \mathbf{B}=0
$$

where $\mathbf{F}$ and $\mathbf{H}$ are the electric and magnetic field intensities, respectively. [Note: we have used " $\mathbf{F}$ " to denote the laser light electric field here rather than the customary notation " $\mathbf{E}$ " to avoid confusion, i.e., "E" and its components denote the (enhanced) atmospheric field at the sensor antenna plate as suggested in section 1 above and in the discussion that follows.] The vector $\mathbf{J}$ is current density, $\rho$ denotes charge density, and we have the relations $\mathbf{D}=\overrightarrow{\boldsymbol{\varepsilon}} \cdot \mathbf{F}$ and $\mathbf{B}=\mu \mathbf{H}$. The electric permittivity $\vec{\varepsilon}$ is a tensor for anisotropic materials; it may be brought into diagonal form by a suitable rotation of coordinates. We will approximate $\mu$ by $\mu_{o}$, the magnetic permeability of a vacuum, for all materials in the optical circuit.

Energy conservation is described by

$$
\int_{S} d a \hat{\mathbf{n}} \cdot(\mathbf{F} \times \mathbf{H})=-\frac{\partial}{\partial t} \int_{d} d v(\mathbf{F} \cdot \mathbf{D}+\mathbf{H} \cdot \mathbf{B}) / 2-\int_{d} d v \mathbf{F} \cdot \mathbf{J},
$$

where $\hat{\mathbf{n}}$ is the outward normal to a surface $S$ which bounds a volume $v$. We will assume that $\rho$ and $\mathbf{J}$ are both zero. The equation for energy conservation can be used to show that the permittivity tensor is symmetric.

Suitable manipulations of (1) for isotropic media yield a wave equation

$$
\left(\nabla^{2}+k^{2}\right) \mathbf{F}=0
$$


The same equation is satisfied by $\mathbf{H}$. Plane wave solutions $\hat{\mathbf{p}} \boldsymbol{e}^{i \mathbf{k} \cdot \mathbf{r}-i \omega t}$ are admitted, where $\hat{\mathbf{p}}$ is a polarization, $\mathbf{k}=k \hat{\mathbf{k}}, k=2 \pi / \lambda$, and $\hat{\mathbf{k}}$ is the direction of propagation. Angular frequency is denoted by $\omega, t$ is time, $\mathbf{r}$ is a position vector, and $\lambda$ is the wavelength. With such assumed plane wave forms for $\mathbf{F}$ and $\mathbf{H}$ in anisotropic media, we have the relations $\mathbf{H}=(\mathbf{k} \times \mathbf{F}) /(\omega \mu)$ and $\mathbf{D}=-(\mathbf{k} \times \mathbf{H}) / \omega$ from which we may obtain (see [17])

$$
(\mathbf{k} \cdot \mathbf{F}) \mathbf{k}-k^{2} \mathbf{F}=-\omega^{2} \mu \ddot{\varepsilon} \cdot \mathbf{F} .
$$

We let $k=\omega n / c$, where $n$ is the wave index of refraction and $c$ is the speed of light, and refer to a coordinate system in which $\vec{\varepsilon}$ is diagonal. The form in (4) is that of an eigenvalue equation. In general, there will be two nontrivial solutions.

The following coordinate system will be used. The $Z$-coordinate will be along the path of the collimated laser beam with the direction from the laser to the photodetector considered as positive. The zenith direction vertical to the optics breadboard, on which the components are mounted, will define $Y$, and $X$ is chosen to complete a right-handed system. Furthermore, we define a system $(x, y, z)$ that is identical to the $(X, Y, Z)$ system but is attached to the crystal; the $(x, y, z)$ system defines the crystal principal axes when no voltage is placed across the crystal. In the present discussion of perfectly aligned optics, there will be no distinction between the two coordinate systems. Later however, in our discussion of misaligned optics provided in section 4 , the $(x, y, z)$ system, or "crystal system", will be oriented differently than the fixed $(X, Y, Z)$ "breadboard system".

The indices of refraction and their associated allowable polarizations may be obtained geometrically. By considering surfaces of constant energy density, an index ellipsoid

$$
x^{2} / n_{x}{ }^{2}+y^{2} / n_{y}{ }^{2}+z^{2} / n_{z}{ }^{2}=1
$$


may be constructed [11]; $n_{x}, n_{y}$, and $n_{z}$ are the refractive indices along the coordinate axes.

The allowable polarizations and indices of refraction may be obtained by finding the axes of the ellipse formed by the intersection of the index ellipsoid and a plane normal to the direction of propagation.

The index ellipsoid of the crystal may be modified by applying an electric field to the crystal. The general formula is given by [11]

$$
\begin{gathered}
\left(n_{x}^{-2}+r_{1 k} e_{k}\right) x^{2}+\left(n_{y}^{-2}+r_{2 k} e_{k}\right) y^{2}+\left(n_{z}^{-2}+r_{3 k} e_{k}\right) z^{2} \\
+2 r_{4 k} e_{k} y z+2 r_{5 k} e_{k} x z+2 r_{6 k} e_{k} x y=1
\end{gathered}
$$

where the summation convention has been employed. The $e_{k}$ are components of the applied electric field, and the $r_{i k}$ are the electro-optic coefficients of the crystal. It is intended that the modulating field be along $\hat{\mathbf{z}}$; only $r_{63}$ is needed since $r_{j 3}=0,1 \leq j \leq 5$ for KDP.

We assume that the crystal is aligned so that its axes of symmetry are parallel to the coordinate axes. The KDP crystal is uniaxial which means that $n_{x}=n_{y} \equiv n_{o}$, where $n_{o}$ has a numerical value of 1.507 and is called the ordinary index of refraction. The extraordinary index of refraction is defined by $n_{e} \equiv n_{z}=1.467$. Since $n_{e}<n_{o}$ the KDP is additionally specified as being a negative uniaxial crystal.

When a field $e_{z}$ is applied along the $z$-direction, the allowed polarizations are $(-\hat{\mathbf{x}}+\hat{\mathbf{y}}) / \sqrt{2}$ and $(\hat{\mathbf{x}}+\hat{\mathbf{y}}) / \sqrt{2}$. Light polarized along $\hat{\mathbf{y}}$ upon entering the crystal is split into these polarizations, the waves along which propagate at different speeds. For a crystal of length $d$ having a voltage $V=e_{z} d$, the phase lag is

$$
\Gamma=\frac{\omega}{c}\left(n_{s}-n_{f}\right) d \cong \frac{2 \pi}{\lambda} n_{\circ}^{3} r_{63} V
$$


where $n_{s}$ (slow) and $n_{f}$ (fast) are the indices of refraction along the allowed polarizations. Waves traveling along the $z$-axis with no modulating voltage have a speed corresponding to the ordinary index. Waves traveling in the $x y$ plane with polarization in the $z$-direction have a speed determined by the extraordinary index of the crystal. The crystal's utility lies in its ability to introduce a variable phase retardation between the two waves in response to an applied voltage. The linear relationship provided in (7) is the Pockels effect [18].

If the laser light field immediately following the first polarizer is $F_{0} \cos \omega t \hat{\mathbf{Y}}$, then the field of the output beam will be

$$
\mathbf{F}=F_{0}[\cos (\omega t-\Gamma)+\cos \omega t] \hat{\mathbf{X}} / 2
$$

Here, the phase change due to the path length between optical components is suppressed. Reflection losses at the interfaces are relatively small, and are customarily neglected. Exact solutions that account for such losses are available ([19], [20]).

Using (8) to obtain the square magnitude $F^{2}$, the power is obtained by taking the time average of $F^{2}$ [21]. Dividing by the input power, which is proportional to $F_{0}^{2} / 2$, yields the optical transmission $T$ given by

$$
T=\sin ^{2} \Gamma / 2=\sin ^{2}\left[(\pi V) /\left(2 V_{\pi}\right)\right]
$$

where $V_{\pi}=\lambda /\left(2 n_{o}^{3} r_{63}\right)$ is called the half-wave voltage, i.e., $V=V_{\pi}$ yields a retardation of $\pi$ which is one half of $2 \pi$, or "one half wave".

Note that the derivative, $T^{\prime}(V) \equiv d T / d V$, or electro-optic sensitivity, is zero when there is no voltage across the crystal, i.e., $T^{\prime}(0)=0$. In order to have the largest possible change in transmission with applied voltage for small voltages, it is convenient to start at a point where $T^{\prime}(0)$ is as large as possible. This may be achieved by introducing an additional phase 
retardation of $\pi / 2$ by inserting a quarter wave plate before the second polarizer. In such a case, the transmission of the system is given by

$$
T=\sin ^{2}[\Gamma / 2+\pi / 4]
$$

This process is the optical analog of biasing an electronic component.

The preceding discussion has summarized the basics of the linear electro-optic effect in anisotropic media. Some early experimental investigations of this effect are provided in [22]. Note from (9) and (10) that a high frequency modulation voltage can be applied to the crystal to vary the optical transmission and hence the optical amplitude of the output beam. In this sense the Pockels cell configuration can serve as a high speed optical shutter.

However, the Pockels cell can also be used in the reverse sense, that is, a high gain measurement of small changes in the optical amplitude of the output beam can be used to infer the changes in crystal voltage. This is the operational principle of the electro-optic lightning detector developed in this work. The lightning places a transient voltage across the electro-optic crystal thereby changing the optical transmission of laser light through the cell. Specific details of the sensor follow.

\section{Sensor Description}

Figure 2 provides a simplistic overview of the electro-optic lightning detector developed at NASA MSFC. The detector is placed outside on a flat surface sufficiently far from tall grass or pointed objects that could produce local field-limiting corona discharges. Note that a thunderstorm separates positive and negative charges and thereby generates strong electric fields in the atmosphere and on the ground. When a cloud-to-ground flash occurs, as shown in Figure 2 , tens of coulombs of negative charge from the lower regions of the thundercloud (i.e., $\sim 7 \mathrm{~km}$ altitude) are deposited to Earth. The large charge transfer is accompanied by a substantial change 
in the electric field $\Delta E_{\mathrm{g}}$ at the ground, i.e., a $\Delta E_{\mathrm{g}}>5 \mathrm{kV} / \mathrm{m}$ is not uncommon for discharges that are within a range of a few kilometers. Those lightning events that do not transfer charge to ground but transport charge wholly within the atmosphere, called cloud flashes, also produce substantial values of $\Delta E_{g}$. In general, the field change at the height of the antenna plate differs from that at the ground, and the presence of the sensor itself enhances the local field change. Figure 2 indicates the net field change at the antenna plate as $\Delta E$, and this is the stimulus that produces a change in crystal voltage given by $\Delta V=\Delta e_{z} d$.

When the sensor detects the lightning-caused field change $\Delta E$, the photocurrent output from the optical system is converted to an analog voltage using a Stanford Research Systems, Inc. current preamplifier (model SR570), and the analog voltage is passed to a Pentium computer where the signal is digitized at $125 \mathrm{~Hz}$ using a Data Translation A/D board (model DT2833).

A closer look at the sensor's optical system is provided in Figure 3(a); it is based on the linear Pockels cell configuration discussed in section 2 . The totality of optics are mounted on an optical breadboard that is $30.48 \mathrm{~cm}$ by $60.96 \mathrm{~cm}$ in dimension. The intent was to build a reasonably small engineering model that still provided "on-line" adjustability and interchangeability of optical components. Future designs of the sensor would be scaled down considerably (i.e., the laser and all optical elements could be precision mounted within a single sealed tube only a few centimeters long and wide).

Working from left to right in Figure 3(a), the first element is a $30 \mathrm{~mW}$ semiconductor laser diode that has a wavelength of $685 \mathrm{~nm}$ and an elliptic beam cross section (major and minor axes of $4 \mathrm{~mm}$ and $1 \mathrm{~mm}$, respectively). We have also used a $4 \mathrm{~mW}, 670 \mathrm{~nm}$ laser diode, but at the expense of reduced sensor sensitivity. The beam height above the optical breadboard is $75 \mathrm{~mm}$ and the beam divergence is negligible across the length of the breadboard. The only considerations during operation are to allow the laser a few minutes of "on time" prior to taking 
data to assure power stability, prevent reflections back into the laser aperture, and prevent excessive temperature changes in the air surrounding the laser.

The next (optional) optic is a beamsplitter that sends a portion of the laser light to a photodetector (shown in the background). These two components allow one to monitor the laser stability, but because our Melles Griot, Inc. lasers had minimal $(<1 \%)$ power fluctuation, the monitoring system was not a strict requirement.

The next element is a post-mounted, vertically oriented Melles Griot dichroic sheet polarizer followed by the KDP crystal. The polarizer has a clear aperature of $26.5 \mathrm{~mm}$ and a thickness of $2.5 \mathrm{~mm}$. The hygroscopic and water soluble KDP crystal is housed in a dry nitrogenflushed air- tight Plexiglass container that is mounted on a two-axis holder; the holder has alignment micrometer adjustments in both yaw and pitch. A durable strap fastens the crystal/container to the holder and the holder is bolted to the optical breadboard. The crystal has a cylindrical geometry ( $40 \mathrm{~mm}$ length, $20 \mathrm{~mm}$ diameter) with ring electrodes wrapped around the outer crystal diameter at each end. The ring electrodes allow for a larger crystal aperture than commonly used washer-type electrodes that are placed directly on the crystal entrance/exit faces. Additional details and considerations of ring electrodes are provided in [23]. Direct measurements of crystal impedance and capacitance give the values $R=194 \mathrm{G} \Omega$ and $C=5.51$ $\mathrm{pF}$, respectively, or a crystal $R C$ decay time of $\sim 1 \mathrm{~s}$. (In addition, we have also experimented with a post-mounted $3 \mathrm{~mm}$ thick, $25.4 \mathrm{~mm}$ aperture crystal of potassium didueterium phosphate $\left(\mathrm{KD}^{*} \mathrm{P}\right)$ similar to the crystal used in [24] and [25]. This crystal had transparent indium tin oxide (ITO) electrodes, and, because of its thin width it had a shorter $R C$ decay time constant than the long cylindrical crystal of KDP.)

The next two optical elements include a quarter wave plate and a horizontal polarizer. Except for its orientation, the polarizer is identical to that described above. The quarter wave plate is composed of quartz and has a diameter of $20 \mathrm{~mm}$. As discussed in section 2, the quarter 
wave plate changes the transmission profile $T(V)$ from that given in (9) to that given in (10) when the optics are perfectly aligned and reflections are negligible. In effect, the quarter wave plate improves the electro-optic sensitivity $T^{\prime}(V)$ at a crystal voltage $V=0$. This is important since, before a lightning event, the voltage on the crystal is very nearly zero (very small and random stray voltages undoubtably exist across the crystal at all times). A subsequent change in crystal voltage $\Delta V$ due to the lightning produces a change in optical transmission given by $\Delta T \cong T^{\prime}(0) \Delta V$ for $\Delta V$ small. Hence, in order to get large (measurable) changes in transmission, it is desirable to have $T^{\prime}(0)$ large. As will be detailed in section 4 , we have found that small misalignments in the optics produce sufficiently large values of $T^{\prime}(0)$ when the quarter wave plate is not inserted.

The final component shown in Figure $3 \mathrm{a}$ is the photodetector. It has an active detector area of $100 \mathrm{~mm}^{2}$ and was connected directly to the input of the SR570 current preamplifier with a short $(30.5 \mathrm{~cm}) 50 \Omega$ plenum coaxial cable. The amplifier (not shown) has a low end sensitivity of 1 volt output per $1 \mathrm{pA}$ current input (written as $1 \mathrm{pA} /$ volt). It also has valuable input offset adjust buttons that allowed us to accurately null the transmitted laser light (at $V=0$ ) so we could increase amplifier gain without over ranging. We typically collected lightning data at either the 1 $\mu \mathrm{A} /$ volt or $50 \mathrm{nA} /$ volt gain setting, depending on storm range. The amplifier also offered considerable band pass filtering capabilities. Lightning data were collected using the $10-30 \mathrm{~Hz}$ band-pass filter, primarily to avoid dc drift and 60-cycle noise sources. Application of the bandpass filter contributed to shortening the overall, or "effective $R C$ " decay time constant of the sensor to a value of tens of milliseconds. The specific value of the effective $R C$ decay depended on the particular amplifier gain setting that was chosen (see section 5 below for additional comments on sensor $R C$ decay and specific examples). 
Figure 3(b) shows the protective housing that the optical system is placed within. The housing is constructed of a $50.8 \mathrm{~mm}$ thick polypropylene material that affords very good thermal insulation from excessive heating due to typical summer conditions in Alabama. Ample room inside the box allowed for the insertion of cooling packs as an added measure to prevent excess heating to the laser and crystal; details of KDP refractive index changes with temperature are provided in [26]. Methods more sophisticated than applying cooling packs are the so-called "quenching conditions" discussed in [27] that remove unwanted birefringences in crystals affected by general changes in environmental conditions. Fortunately, under good thunderstorm conditions the weather was cloudy and not excessively hot, particularly during periods of precipitation. In these situations, we saw no discernable difference in sensor output with or without cooling packs.

The polypropylene material had sufficient weight and shock absorption to minimize the effects of vibrations due to wind/precipitation/thunder that might disturb the optical alignment. Great care was spent assuring that the box was watertight; the box top lid overhangs the sides and a watertight $\mathrm{O}$-ring lines the area where the lid rests. The outside of the box was coated with a durable water-repellent paint and the color was white to reflect sunlight (again preventing excessive heating inside the box). The inside of the box was coated with a flat black paint to minimize scattering of laser light that might be reflected from optical elements.

On the outside top portion of the box lid is mounted a teflon rod that supports a circular aluminum antenna plate. A small bore hole was drilled through the teflon rod so that a tefloncoated wire could inconspicuously connect the antenna plate to the left ring electrode of the crystal. The other crystal ring electrode was grounded to the optical breadboard, as was the current preamplifier. The optical breadboard and aluminum ground plane (mounted on top of the box lid) were both connected to a standard $8 \mathrm{ft}$ copper ground rod that was driven into the Earth a few inches from the housing. A small teflon "rain cap" was placed over the teflon support rod 
before bolting the antenna plate down; this prevents streams of water from shorting the antenna to ground potential.

In summary, the lightning-caused $\Delta E$ excites the sensor antenna and raises it to a transient voltage, $\Delta V$, measured between the antenna plate (the left-side crystal electrode) and ground (the right-side crystal electrode); here we have neglected the small capacitance between the antenna plate and the left crystal electrode. The voltage $\Delta V$ across the crystal changes the crystal's refractive index and polarization characteristics, and hence the transmission of the optical system changes by an amount $\Delta T$. With laser power $P_{o}$, the change in transmitted power is simply $\Delta P=\Delta T P_{o}$ which is measured using a high gain photodetector/preamplifier system. Therefore, one formally has the chain $\Delta E \rightarrow \Delta V \rightarrow \Delta T \rightarrow \Delta P$ so that values of $\Delta P$ can be directly related to the values of $\Delta E$ through calibration (see section 5 below).

\section{Alignment}

The expressions for the optical transmission, $T$, given in (9) and (10) assume that the optical elements are perfectly aligned. Since in this study the Pockels cell is used as a detector to sense the slightest antenna voltages, we have found that even small misalignments of the crystal and/or quarter wave are important effects.

The theory of transmission through birefringent media is well developed and the transmission through a crystal at an oblique angle can be derived by exact or approximate means ([19], [20], [28], [29]). When a collimated beam of light enters a birefringent medium at an oblique angle, the transmitted field consists of two beams that propagate in two distinct directions. The direction of a refracted beam depends on its angle of incidence and the refractive index, and the refractive index in turn depends on the angle of incidence. An exact solution to this problem is complicated, but a great simplification can be made by noting that there is very 
little divergence in the transmitted beams, particularly for small angles of incidence. We will assume that the transmitted field consists essentially of a single beam whose direction is determined by the ordinary index of refraction.

As defined in section 2 , the crystal axes $(x, y, z)$ are parallel to the breadboard axes $(X, Y, Z)$ when the optics are perfectly aligned. Suppose that the crystal is misaligned by an angle $-\Theta$ about an axis $\hat{\mathbf{h}}=-\sin \phi \hat{\mathbf{X}}+\cos \phi \hat{\mathbf{Y}}$, where $\phi$ is measured from the positive $X$-axis. Inside the crystal, both refracted rays are assumed to make an angle $\theta$ with the crystal's extraordinary axis, where $\sin \Theta=n_{0} \sin \theta$. A formula for retardation may be obtained by examining the index ellipsoid.

The equation for the index ellipsoid and expressions for related variables can be summarized as

$$
\mathbf{r}^{T} \cdot \overrightarrow{\mathbf{N}} \cdot \mathbf{r}=1, \quad \mathbf{r}=x \hat{\mathbf{x}}+y \hat{\mathbf{y}}+z \hat{\mathbf{z}}, \quad \overline{\mathbf{N}}=\left(\begin{array}{ccc}
n_{o}^{-2} & \varepsilon & 0 \\
\varepsilon & n_{o}^{-2} & 0 \\
0 & 0 & n_{e}^{-2}
\end{array}\right), \quad \varepsilon=e_{z} r_{63}
$$

Ideally, light should pass along $\hat{\mathbf{z}}$; however, in the misaligned case the optical path is along $\sin \theta(\cos \phi \hat{\mathbf{x}}+\sin \phi \hat{\mathbf{y}})+\cos \theta \hat{\mathbf{z}}$. Such a direction may be obtained by rotating $\hat{\mathbf{z}}$ about $\hat{\mathbf{h}}$ through an angle $\theta$. This may be done with a rotation matrix whose components are obtained from the dyadic

$$
\ddot{\mathbf{M}}=[\overline{\mathbf{I}}-(1-\cos \theta)(\overrightarrow{\mathbf{I}}-\hat{\mathbf{h}} \hat{\mathbf{h}})+\sin \theta \hat{\mathbf{h}} \times \overrightarrow{\mathbf{I}}]
$$

where $\overrightarrow{\mathbf{I}}$ is the identity dyadic. In a rotated system, the equation of the index ellipsoid and related variables are given by

$$
\mathbf{r}^{\prime T} \cdot \overrightarrow{\mathbf{N}}^{\prime} \cdot \mathbf{r}^{\prime}=1, \overrightarrow{\mathbf{M}} \cdot \mathbf{r}^{\prime}=\mathbf{r}, \quad \overrightarrow{\mathbf{N}}^{\prime}=\overrightarrow{\mathbf{M}}^{T} \cdot \overrightarrow{\mathbf{N}} \cdot \overrightarrow{\mathbf{M}}
$$

and the optical path becomes $\hat{\mathbf{z}}^{\prime}$. 
Polarizations and indices of refraction may be obtained from the components of $\overrightarrow{\mathbf{N}}_{2}^{\prime}$ given by:

$$
\begin{gathered}
\overrightarrow{\mathbf{N}}_{2}^{\prime}=\left(\begin{array}{cc}
\alpha & \beta \\
\beta & \gamma
\end{array}\right) ; \\
\alpha=\left(A_{1}^{2}+A_{2}^{2}\right) n_{o}{ }^{-2}+A_{3}^{2} n_{e}{ }^{-2}+2 A_{1} A_{2} \varepsilon \\
\beta=A_{2}\left(A_{1}+A_{4}\right) n_{o}{ }^{-2}+A_{3} A_{5} n_{e}{ }^{-2}+\left(A_{1} A_{4}+A_{2}^{2}\right) \varepsilon \\
\gamma=\left(A_{2}^{2}+A_{4}^{2}\right) n_{o}^{-2}+A_{5}^{2} n_{e}^{-2}+2 A_{2} A_{4} \varepsilon ; \\
A_{1}=\cos \theta \cos ^{2} \phi+\sin ^{2} \phi, A_{2}=(\cos \theta-1) \cos \phi \sin \phi, A_{3}=\sin \theta \cos \phi, \\
A_{4}=\cos \theta \sin ^{2} \phi+\cos ^{2} \phi, A_{5}=\sin \theta \sin \phi,
\end{gathered}
$$

where the intersection to the plane normal to the beam direction and the index ellipsoid is the ellipse $\mathbf{r}_{2}^{\prime T} \cdot \overrightarrow{\mathbf{N}}_{2}^{\prime} \cdot \mathbf{r}_{2}^{\prime}=1$ and $\mathbf{r}_{2}^{\prime}=x^{\prime} \hat{\mathbf{x}}^{\prime}+y^{\prime} \hat{\mathbf{y}}^{\prime}$. The refractive indices and allowed polarizations may be obtained from the eigenvalues and eigenvectors of this matrix. Performing these calculations yields the two eigenvalues

$$
\begin{aligned}
\lambda_{ \pm}= & \frac{1}{2}\left\{2 n_{o}{ }^{-2}+\sin ^{2} \theta n-\sin ^{2} \theta \sin 2 \phi \varepsilon\right. \\
& \pm\left[\sin ^{4} \theta n+2\left(1+\cos ^{2} \theta\right) \sin ^{2} \theta \sin 2 \phi n \varepsilon\right. \\
& \left.\left.+\left(\sin ^{4} \theta \sin ^{2} 2 \phi+4 \cos ^{2} \theta\right) \varepsilon^{2}\right]^{1 / 2}\right\} \\
& \approx n_{o}{ }^{-2}+\theta^{2}(n-\sin 2 \phi \varepsilon) / 2 \pm\left[\theta^{4} n^{2} / 4+\theta^{2} \sin 2 \phi n \varepsilon+\varepsilon^{2}\right]^{1 / 2}
\end{aligned}
$$

for small $\theta$, where $n=n_{e}{ }^{-2}-n_{o}{ }^{-2}$. The retardation is $\Gamma=2 \pi d \sec \theta \Delta / \lambda$, where

$$
\Delta=n_{s}-n_{f}=1 / \sqrt{\lambda_{-}}-1 / \sqrt{\lambda_{+}} \approx n_{o}^{3} \sqrt{\theta^{4} n^{2} / 4+\theta^{2} \sin 2 \phi E+E^{2}} .
$$

Eigenvectors are given by

$$
\hat{\mathbf{v}}_{-}^{\prime}=\cos \delta \hat{\mathbf{x}}^{\prime}-\sin \delta \hat{\mathbf{y}}^{\prime}, \quad \hat{\mathbf{v}}_{+}^{\prime}=\sin \delta \hat{\mathbf{x}}^{\prime}+\cos \delta \hat{\mathbf{y}}^{\prime}
$$

where $\tan 2 \delta=2 \beta /(\gamma-\alpha)$. The + and - signs indicate the fast and slow axes, respectively. Consistent with the approximations used, the field exiting the second polarizer has the form 


$$
\begin{gathered}
F_{0}\left[\left(\hat{\mathbf{V}}_{+} \cdot \hat{\mathbf{X}}\right)\left(\hat{\mathbf{V}}_{+} \cdot \hat{\mathbf{Y}}\right) \cos \omega t+\left(\hat{\mathbf{V}}_{-} \cdot \hat{\mathbf{X}}\right)\left(\hat{\mathbf{V}}_{-} \cdot \hat{\mathbf{Y}}\right) \cos (\omega t-\Gamma)\right] \hat{\mathbf{X}} \\
\hat{\mathbf{V}}_{-}=\cos \delta \hat{\mathbf{X}}-\sin \delta \hat{\mathbf{Y}}, \hat{\mathbf{V}}_{+}=\sin \delta \hat{\mathbf{X}}+\cos \delta \hat{\mathbf{Y}} .
\end{gathered}
$$

Obtaining the transmission as for (9), we have

$$
T=\sin ^{2} 2 \delta \sin ^{2} \Gamma / 2,
$$

where $\sin 2 \delta=2 \beta / \sqrt{(\alpha-\gamma)^{2}+4 \beta^{2}}$. If a quarter wave plate is used, similar reasoning leads to the formula

$$
T=\frac{1}{2}[1+\sin 2 \delta \sin \Gamma]
$$

The choice of sign for the square root corresponds to the quarter wave plate installed in such a way as to increase transmission for increasing voltage $\left(e_{z}\right.$ being taken to be positive in the positive direction of the $Z$-axis).

In order to provide for a most general misalignment of the crystal, we allow for a rotation of the crystal about $\hat{\mathbf{Z}}$ by an angle $\tau$. Results may be obtained by rotating the orientations of the polarizers and quarter wave plate by $-\tau$. Transmissions in this case are given by (19) and (20) with $\delta$ replaced with $\delta+\tau$.

Using the expressions in (19) and (20), we have computed the theoretical transmission of an aligned and misaligned optical system with or without a quarter wave plate. The results are provided in Figure 4. We have estimated the small misalignments expected under normal laboratory conditions, i.e., $\theta=1.4^{\circ}$ so that $\Theta=\sin ^{-1}(1.5074 \sin 1.4)=2.11^{\circ}$ about an axis $\hat{\mathbf{h}}$ oriented with angle $\phi=89.75^{\circ}$.

Note that when there is no quarter wave plate and the optics are aligned (19) reduces to (9) and a simple sine squared dependence results (solid line, Figure 4(a)). When the optics are misaligned, the net transmission increases slightly, but more importantly, the sensitivity $T^{\prime}(V)$ increases substantially at and near $V=0$ (dashed line, Figure $4(a)$ ). When the quarter wave is 
inserted, the transmission increases for both aligned (dotted line, Figure $4(b)$ ) and misaligned (dot-dashed line, Figure 4(b)), but the misalignment no longer improves sensor sensitivity at and near $V=0$, or at any larger values of $V$.

To further clarify the variation of sensor sensitivity for small changes in crystal voltage, we have numerically computed the change in transmission $\Delta T=T(V)-T(0)$ for a small crystal voltage change $\Delta V=V-0=V$ all as a function of $V$. The functional dependence $\Delta T=f(V)$ is provided in Figure 5 for aligned and misaligned optics with or without a quarter wave plate. We see that the addition of the quarter wave plate necessarily improves sensor sensitivity no matter if the system is aligned or misaligned. However, because the quarter wave plate produces additional reflective losses to the optical system, because its inclusion produces additional noise sources during high-gain operation of the lightning detector, and because the slope of the lines in Figure 5(b) have magnitudes that are not vastly different, we did not include the use of the quarter wave plate during normal calibration and operation of the lightning sensor.

\section{Calibration}

The calibration of the lightning detector was straightforward. On the top lid of the optical housing shown in Figure 3a, four threaded holes were drilled (one at each corner of the square lid). A nylon rod was screwed into each hole in order to support a large circular aluminum calibration plate. An Avtech high voltage wave function generator (HVWFG) was connected between the calibration plate and the lid-mounted ground plane. This provided a uniform vertical electric field change for the lightning detector antenna plate located between the calibration plate and ground plane. We also used a standard (i.e., lower voltage) BK-Precision WFG to check low-end sensor detectability. 
The risetime of a lightning return stroke field change is typically $60 \mu$ s. The Avtech HVWFG could supply square waves having amplitudes of \pm 500 volts with frequencies ranging from near dc to $3 \mathrm{kHz}$ and with a peak-to-peak risetime $<35 \mu \mathrm{s}$. The BK Precision WFG could supply square waves having amplitudes of \pm 12 volts with frequencies ranging from near dc to several megahertz, and a risetime of about $2 \mathrm{~ms}$ when operated at $0.5 \mathrm{~Hz}$. In order to get an accurate calibration, it is important to have waveforms that rise to peak in milliseconds or less because of the characteristic $R C$ decay of the crystal. In effect, the vertical displacement current density $\varepsilon_{0} d E / d t$ produced by the calibration apparatus or a lightning event must be large enough to place a significant amount of charge onto the left crystal electrode before leakage currents in the crystal drain the crystal voltage to ground. The electric field line distribution inside an electro-optic parallelepiped crystal is provided in [30] and evidence for field-driven leakage currents in electro-optic materials is given in [3] and [24].

Because we have picked a crystal that has an $R C$ decay of $\sim 1 \mathrm{~s}$, the leakage current to ground is slow. This allows the crystal to acquire a larger voltage, and hold a voltage for a longer period of time. Furthermore, since electro-optic crystals can be modulated into the gigahertz region, they respond quickly to changes in voltage. Hence, the crystal responds quickly to lightning transients, acquires a sufficient voltage, and holds the voltage for a time that is long in comparison to the (tens of milliseconds) decay time of the photodetector/amplifier system. The amplifier effectively samples the crystal voltage substantially faster than the crystal voltage has a chance to decay, thereby optimizing overall sensor sensitivity.

If it were not for our desire to remove 60-cycle noise sources, we could replace the applied band-pass filter with a high-pass filter $(>10 \mathrm{~Hz})$ to improve overall sensor bandwidth. A narrow filter for removing 60-cycle noise in conjunction with the high-pass filter would clearly be optimum, but is not possible with the SR570 electronics alone. 
Figure 6 shows some specific examples of sensor output in response to both high and low voltage WFG-simulated lightning electric field changes; the amplifier gain was set to $1 \mu \mathrm{A} /$ volt and the $10-30 \mathrm{~Hz}$ band-pass filter was used. The train of sensor output pulses provided in Figure $6(\mathrm{a})$ are due to $\mathrm{a} \pm 2083 \mathrm{~V} / \mathrm{m}$ square wave calibration input; this field change magnitude is typical of lightning that is a few kilometers away from the observer. Note that the output waveforms decay back to zero with an e-folding time (effective $R C$ decay) of $\sim 14 \mathrm{~ms}$. The amplifier then "overshoots" until it recedes back completely to zero about $154 \mathrm{~ms}$ later. At a gain of $50 \mathrm{nA} /$ volt, there was no overshooting and the e-folding time was $\sim 27 \mathrm{~ms}$.

Figure 6(b) shows the output for a square wave input of only $\pm 30.6 \mathrm{~V} / \mathrm{m}$. The low-end sensitivity and signal-to-noise shown in Figure $6(\mathrm{~b})$ can be further improved by further optimizing the misalignment of the optical system. Additional details related to the general accuracy and limits of precision of electro-optic voltage sensors is provided in the U.S. National Bureau of Standards (NBS) report given in [31].

We performed these type of calibration tests for a variety of simulated lightning $\Delta E$ values and amplifier gains when the sensor was deployed outside of building 4614 at the NASA MSFC. Figure 7 shows a sample linear response calibration plot acquired over a large range of $\Delta E$ values when the gain was set to $1 \mu \mathrm{A} /$ volt. Because the KDP crystal half wave voltage $V_{\pi}$ is about 9000 volts, the lightning detector's full dynamic range well exceeds that shown in Figure 7 . In addition, because of the misalignment of optical elements, the value of $\delta$ in (19) differs from $\pi / 4$ so that we are able to discriminate between positive and negative lightning $\Delta E$ s, i.e., the linear calibration curve extends to negative values of $\Delta E$.

\section{Storm Data}


The summer of 1998 in northern Alabama was marked by substantially dry periods that led to eventual fire outbreaks in September. Nonetheless, we were able to deploy the lightning detector on five separate occasions for storms that occurred on June 30, and July 3, 20, 21, and 24. The storm occurring on July 20 resulted in a lightning death to a citizen of northern Huntsville, Alabama that occured about 45 minutes prior to the start of our data collection period.

The National Lightning Detection Network (NLDN) operated by Global Atmospherics Inc. (GAI) located in Tucson, Arizona detected widespread cloud-to-ground activity on June 30 over much of northern Mississippi, Alabama, and Georgia, and over most of Tennessee. Several cloud-to-ground lightning events that occurred within a few kilometers of our deployment site were identified by eye and were time-correlated with transient sensor outputs that we displayed in real time on the Pentium computer. We used Microcal Origin 4.0 data acquisition software in "scope" mode to look at the digitized sensor output. We also viewed the direct analog output of the sensor on a conventional $40 \mathrm{MHz}$ oscilliscope. Our sensor detects both ground and cloud discharges, but for these daytime thunderstorms it was more practical to time-correlate events with ground discharges, i.e., it was easier to see the well-defined channel of a ground stroke rather than the faint diffuse emission from cloud-obscured discharges.

Figure 8(a) shows the output from the electro-optic lightning detector for an active 2-min period during the June 30 storm (the plot abscissa is in seconds after 4:48:30.5 PM CST, i.e., the time the sensor was turned on). These data were collected using a gain of $50 \mathrm{nA} /$ volt, or a factor of 20 higher than that shown in the calibration curve in Figure 7. By looking southward from our deployment site during this storm, we were able to visually confirmed over 10 lightning events that gave coincident field excursions in our recorded sensor output. Two small amplitude lightning flashes are indicated with pointers in Figure 8(a), and a more time-resolved plot of each 
flash is provided in Figure 8(b) and 8(c). These particular flashes were confirmed by eye as cloud-to-ground events. Several rapid variations are evident in each flash that we believe are individual strokes. The duration between the peaks of these excursions is less than $\sim 100 \mathrm{~ms}$ which is characteristic of the interstroke time interval shown in Figure 3 of [2]. There may even be evidence of a small amplitude " $\mathrm{K}$ " change just after $3160.4 \mathrm{~s}$ in Figure $8(\mathrm{c})$. There are some positive excursions starting at $\sim 3122.7 \mathrm{~s}$ in Figure $8(\mathrm{~b})$ that might be associated with stepped leader activity or other local atmospheric electrical effects. We believe that the negative excursion before the series of positive strokes is associated with an upward in-cloud moment change; a similar negative excursion also occurs in the flash shown in Figure 8(c).

As we have stated, vibrations due to wind, precipitation, and thunder can produce noise in the sensor output and these affects are evident for small amplitude discharges. Figure 9 shows some time-resolved, single pulse, large amplitude events that occurred earlier during the June 30 storm. There are several low amplitude reverse-polarity field changes associated with distant lightning events, and two larger amplitude events that are probably much closer to the sensor. It is possible that the reverse-polarity events are associated with cloud discharges that occur beyond the "reversal distance", i.e., the surface field verses range from a tripolar thundercloud charge distribution reverses sign at the "reversal distance", or about $10 \mathrm{~km}[1]$.

The saturation output voltage of the SR570 amplifier is \pm 5 volts which was occasionally exceeded by some lightning events. In some cases, we were forced to decrease sensor gain to avoid frequent pulse saturation in the data stream. An RS232 cable connection between the amplifier and our indoor computer allowed us to remotely adjust sensor gain as the storm cells moved or developed relative to the deployment site. 


\section{Summary}

In the future, we would like to further experiment with and optimize the electro-optic lightning sensor and deploy it again to collect more storm data. As we have stated, the dimensions of our sensor could be scaled down significantly. The laser and all optical elements could be precision-mounted within a single sealed tube only a few inches long and wide. Alternately, a small crystal attached to polarization maintaining (PM) fibers and an antenna could be the sole components deployed into the thunderstorm environment; the photodetector, current amplifier, laser, and remaining optics could all reside inside a building along side the data acquisition computer. Or, crossed polarizers could be mounted to the crystal and regular fiber optic cables used. In any case, a single unit reduced size system would be less susceptible to vibration-induced noise sources.

Size reduction, as well as improvements in low end sensitivity and signal-to-noise are fundamental to improving the attractiveness of electro-optic applications in the discipline of lightning research. It is possible to arrange optics in such a way as to pass the laser light multiple times through the crystal to increase the total birefringence and thereby substantially improve sensor gain. We are also interested in inspecting other crystal types that offer certain advantages such as bismuth germanate $\left(\mathrm{Bi}_{4} \mathrm{Ge}_{3} \mathrm{O}_{12}\right)$ described in [32] and Hexamine $\left(\left(\mathrm{CH}_{2}\right)_{6} \mathrm{~N}_{4}\right)$ described in [33].

Ultimately, the electro-optic technology needs to be placed beside a conventional electric field mill such as the type operated by the NASA Kennedy Space Center (KSC) and the U.S. Air Force Eastern Range (ER) located in east central Florida. This would allow one to further inspect the sensitivity, accuracy, signal, and noise characteristics of the electro-optic sensor under a variety of environmental conditions and lightning source stimuli. 
In the future, lightning field changes derived from a ground-based network of electro-optic sensors that cover a region of a few hundred square kilometers could be used to retrieve the charges deposited by a lightning. This has already been achieved using the KSC-ER field mill network, but with the added difficulty and errors associated with detecting and computing values of $\Delta E$ from the continuous $E$-data ([33] - [37]).

Acknowledgments: We are grateful for the assistance of NASA MSFC's computer aided design engineer Mr. Steven Benefield, the graphical art support of Janine Roskowski, and the final manuscript editing performed by Susan Burrer. This work was made possible through NASA Center Director's Discretionary Fund (CDDF) project \#96-11. 


\section{References}

[1] Uman, M. A., The Lightning Discharge, International Geophysics Series, Vol. 39, Academic Press, Inc., Orlando, Florida, 1987.

[2] Kitigawa, N., and M. Brook, A comparison of intracloud and cloud-to-ground lightning discharges, J. Geophys. Res., 65, $1189-1201,1960$.

[3] Osamu, K., and K. Kakishita, Electo-optical effect of $\mathrm{Bi}_{4} \mathrm{Ge}_{3} \mathrm{O}_{12}$ crystals for optical voltage sensors, Jpn. J. Appl. Phys., 32, 4288 - 4291, 1993.

[4] Hilliard, D. P., and D. L. Mensa, Photonic electromagnetic field sensor apparatus, United States Patent \#5243186, September 7, 1993.

[5] Nelson, B. N., C. Menzel, and T. G. DiGiuseppe, Fiber optic electric field sensor configurations for high bandwidth lightning research measurement applications, SPIE: High Bandwidth Analog Applications of Photonics, 720, 85 - 91, 1986.

[6] Masterson K. D., Photonic electric-field probe for frequencies up to $2 \mathrm{Ghz}$ SPIE: High Bandwidth Analog Applications of Photonics, 720, 100 - 104, 1986.

[7] Kanoi, M., G. Takahashi, T. Sato, M. Higaki, E. Mori, and K. Okumura, Optical voltage and current measuring system for electric power systems, IEEE Trans. on Power Delivery, PWRD-1, $91-97,1986$. 
[8] Kyuma, K., S. Tai, M. Nunoshita, N. Mikami, and Y. Ida, Fiber-optic current and voltage sensors using a $\mathrm{Bi}_{12} \mathrm{GeO}_{20}$ single crystal, J. Light. Tech., LT-1, 1983.

[9] Shibata, K., A fibre optic electric field sensor using the electrooptic effect of $\mathrm{Bi}_{4} \mathrm{Ge}_{3} \mathrm{O}_{12}$, Fuji Electric Corporate Research and Development, Ltd., Japan (Presented paper preprint), circa 1982.

[10] Hamasaki, Y., H. Gotolt, M. Katoh, and S. Takeuchi, OPSEF: An optical sensor for measurement of high electric field strength, Elect. Let., 16, $406-407,1980$.

[11] Yariv, A., and P. Yeh, Optical Waves in Crystals, John Wiley \& Sons, New York, 1984.

[12] Tahara, N., M. Shimizu, K. Nakamura, E. Enukai, and Y. Kojima, A lightning warning device using liquid crystal elements, Sixth International Conference on Dielectric Materials (Conf. Pub. No. 363), Sponsored by the Institution of Electric and Electronic Engineers (IEEE) United Kingdom, 397-400, 1992.

[13] Sliker, T. R., and S. R. Burlage, Some dielectric and optical properties of $\mathrm{KD}_{2} \mathrm{PO}_{4}, J . A p p l$. Phys., 34, $1837-1840,1963$.

[14] Pockels, F., Abhandlungen der Gesellschaft der Wissenschaften zu Gottingen, 39, 1, 1893.

[15] Pockels, F., Lehrbuch der Kristalloptik, Leipzig, 1906. 
[16] Jackson, J., Classical Electrodynamics, 2nd Ed., John Wiley \& Sons, New York, p. 218, 1975.

[17] Ghatak, A. and K. Thyagarajian, Optical Electronics, Cambridge Univ. Press, Cambridge, pp. $74-89,1989$.

[18] Levi, L., Applied Optics, a Guide to Optical System Design, Vol. 2, John Wiley \& Sons, New York, pp. 283-284, 1980.

[19] Holmes, D., Exact theory of retardation plates, J. Opt. Soc. Amer., 54, 1115-1120, 1964.

[20] Holmes, D., and D. Freucht, "Electromagnetic wave propagation in birefringent multilayers", J. Op. Soc. Am., 56, 1763-1769, 1966.

[21] Mickleson, A., Physical Optics, Van Nostrand Reinhold, New York, p. 17, 1992.

[22] Billings, B. H., The electro-optic effect in uniaxial crystals of the type $\mathrm{X}_{2} \mathrm{PO}_{4}$, II. Experimental, J. Opt. Soc. Amer., 39, 802 - 807, 1949.

[23] Steinmetz, L. L., T. W. Pouliot, and B. C. Johnson, Cylindrical, ring-electrode KD*P electrooptic modulator, Appl. Opt., 12, 1468 - 1471, 1973.

[24] West, E. A., Using $\mathrm{KD}^{*} \mathrm{P}$ modulators for polarization measurements of the Sun, SPIE: Polarization Considerations for Optical Systems II, 1166, 434 - 445, 1989. 
[25] West, E. A., and N. Wilkins, DC bias moulation characteristics of longitudinal KD*P modulators, SPIE: Polarization Analysis and Measurement, 1746, 386 - 394, 1992.

[26] Phillips, R. A., Temperature variation of the index of refraction of ADP, KDP, and Deuterated KDP, J. Opt. Soc. Amer., 56, 629 - 632, 1966.

[27] Lee, K. S., New compensation method for bulk optical sensors with multiple birefringences, Appl. Opt., 28, $2001-2011,1989$.

[28] Billings, B. H., The electro-optic effect in uniaxial crystals of the type $\mathrm{XH}_{2} \mathrm{PO}_{4}$, I. Theoretical, J. Opt. Soc. Amer., 39, 797 - 801, 1949.

[29] Billings, B. H., The electro-optic effect in uniaxial crystals of the dihydrogen phosphate ( $\mathrm{X}$ $\mathrm{H}_{2} \mathrm{PO}_{4}$ ) type. IV. Angular field of the electro-optic shutter, J. Opt. Soc. Amer., 42, 12 - 20, 1952.

[30] Gilbert, M. A., Polarizability of an electro-optic parallelepiped crystal, SPIE: High Bandwidth Analog Applications of Photonics, 720, 92 - 99, 1986.

[31] Day, G. W., P. D. Hale, M. Deeter, T. E. Milner, D. Conrad, and S. M. Etzel, Limits to the Precision of Electro-Optic and Magneto-Optic Sensors, NBS Technical Note 1307, \#PB87212536, U.S. Department of Commerce, 1987. 
[32] Williams P. A., A. H. Rose, K. S. Lee, D. C. Conrad, G. W. Day, and P. D. Hale, Optical, thermo-optic, electro-optic, and photoelastic properties of bismuth germanate $\left(\mathrm{Bi}_{4} \mathrm{Ge}_{3} \mathrm{O}_{12}\right)$, Appl. Opt., 35, 3562 - 3568, 1996.

[33] Forno, C., and O. C. Jones, Hexamine electro-optic light modulators, J. of Phys. E: Scientific Instruments, 7, $101-$ 104, 1974.

[34] Koshak, W. J., and E. P. Krider, A linear method for analyzing lightning field changes, $J$. Atmos. Sci., 51, 473 - 488, 1994.

[35] Koshak, W. J., and E. P. Krider, Analysis of lightning field changes during active Florida thunderstorms, J. Geophys. Res., 94, 1165-1186, 1989.

[36] Maier, L. M., and E. P. Krider, The charges that are deposited by cloud-to-ground lightning in Florida, J. Geophys. Res., 91, 13,275-13,289, 1986.

[37] Jacobson, E. A., and E. P. Krider, Electrostatic field changes produced by Florida lightning, J. Atmos. Sci., 33, 103-117, 1976. 


\section{Figure Captions}

Figure 1. Typical electro-optic Pockel cell light amplitude modulator [adapted from Yariv and Yeh, 1984].

Figure 2. Overview of the electro-optic lightning detector system; $\Delta E$ denotes a thunderstorm electric field change produced by a cloud-to-ground lightning event shown to the left.

Figure 3. The basic components of the electro-optic lighting detector: (a) optics, and (b) the optical housing and antenna.

Figure 4. Comparison of the transmission $T$ for aligned and misaligned optics (a) without a quarter wave plate and (b) with a quarter wave plate.

Figure 5. The change in transmission $\Delta T$ for a voltage change $\Delta V=V-0=V$ across the crystal all as a function of $V$, with and without a quarter wave plate for (a) aligned optics and (b) misaligned optics.

Figure 6. Some calibration results for (a) a typical lightning field change of $2083 \mathrm{~V} / \mathrm{m}$ and (b) a very small $30.6 \mathrm{~V} / \mathrm{m}$ field change that would be produced by a distant lightning source. The band-pass filter of the amplifier is $10-30 \mathrm{~Hz}$ and the amplifier gain is $1 \mu \mathrm{A} /$ volt. 
Figure 7. Sample calibration curve across a wide dynamic range of $\Delta E$ values. A linear regression fit to the data points shown is $\Delta E=-21.130+371.495 \Delta V$; the linear regression constant is $r=0.99902$. The band-pass filter of the amplifier is $10-30 \mathrm{~Hz}$ and the amplifier gain is $1 \mu \mathrm{A} /$ volt.

Figure 8. Example of lightning data results for a storm that occurred on June 30, 1998: (a) for a 2-min period, (b) for a cloud-to-ground lightning flash within the 2-min period, and (c) another cloud-to-ground flash in the 2 minute period. The band-pass filter of the amplifier is $10-30 \mathrm{~Hz}$ and the amplifier gain is $50 \mathrm{nA} /$ volt, or a factor of 20 higher than that used in the calibration plot of Figure 7.

Figure 9. Additional examples of larger amplitude time-resolved lightning field changes collected from the June 30, 1998 storm. Several small field excursions exist due to distant lightning. The band-pass filter of the amplifier is $10-30 \mathrm{~Hz}$ and the amplifier gain is $50 \mathrm{nA} /$ volt, or a factor of 20 higher than that used in the calibration plot of Figure 7. 